\title{
Mehr Mut in der Rentenpolitik! Ein Vorschlag
}

\author{
In der Öffentlichkeit besteht Konsens darüber, dass das deutsche Rentensystem dringend \\ reformiert werden muss, da das Verhältnis von Beitragszahlern zu Rentnern immer ungünstiger \\ werden wird. Die Regierungskommission „Verlässlicher Generationenvertrag“ hat Ende März \\ 2020 in ihrem Abschlussbericht vorgeschlagen, einen Alterssicherungsbeirat einzuführen. In \\ diesem Beitrag wird gefragt, ob ein unabhängiges Gremium dabei helfen könnte, notwendige \\ Reformen der gesetzlichen Rentenversicherung zu realisieren, ohne dass Politiker deswegen \\ um ihre Wiederwahl fürchten müssen. Der hier entwickelte Vorschlag geht deutlich über den \\ der Rentenkommission hinaus.
}

\begin{abstract}
Die Lebenserwartung der Deutschen steigt stetig - und ab jetzt gehen zudem noch die großen Geburtenjahrgänge der „Babyboomer“ in Rente. Es scheint daher unausweichlich, die Stellschrauben der Rentenversicherung an eine zunehmende Zahl von Rentenempfängern anzupassen. Dazu kann man den Beitragssatz und den Bundeszuschuss erhöhen, das Rentenniveau absenken oder aber das Renteneintrittsalter erhöhen. Keiner dieser Parameter ist unstrittig, und jede Reform fällt schwer. Leichter ist es, immer wieder Beratungskommissionen auf Ad-hoc-Basis einzusetzen, so auch in der laufenden Legislaturperiode mit der Regierungskommission „Verlässlicher Generationenvertrag“ (2020), die Ende März ihren Abschlussbericht vorgelegt hat.
\end{abstract}

Dass dieser Bericht keine eindeutigen Empfehlungen enthält, mag einige Politiker enttäuschen - andere wird es freuen. Damit stellt sich die Frage, ob der Politik mit derartiger Ad-hoc-Beratung wirklich geholfen ist - und genau diese Frage hat sich auch die Rentenkommissi-

(c) Der/die Autor(en) 2020. Open Access: Dieser Artikel wird unter der Creative Commons Namensnennung 4.0 International Lizenz (https:// creativecommons.org/licenses/by/4.0/deed.de) veröffentlicht.

Open Access wird durch die ZBW - Leibniz-Informationszentrum Wirtschaft gefördert.

Prof. Dr. Stefan Voigt ist Direktor am Institut für Recht und Ökonomik der Universität Hamburg und Network Fellow beim CESifo in München. on gestellt. Sie schlägt einen Alterssicherungsbeirat vor, der als „unabhängige und pluralistische Instanz" Gesetzgeber und Regierung beraten soll. Im Bericht wird die konkrete Ausgestaltung des Alterssicherungsbeirats bewusst offen gelassen. Dazu heißt es: „Für die konkrete Ausgestaltung der Berufung, der Arbeitsweise und der Beschlussfassung des Alterssicherungsbeirats sind viele Möglichkeiten denkbar. Die Ausgestaltung sollte erst nach einem offenen Diskussionsprozess gesetzlich geregelt werden.“ (Regierungskommission „Verlässlicher Generationenvertrag", 2020).

\section{Unabhängige Gremien}

In vielen Demokratien gibt es inzwischen eine Vielzahl unabhängiger Gremien, deren Mitglieder über unterschiedlich weitgehende Kompetenzen verfügen und die sich keiner Wiederwahl stellen müssen. Von Majone (1996) wurden sie als "non majoritarian institutions" bezeichnet. Vibert (2007) hat vorgeschlagen, sie „unelected bodies“ zu nennen. Bekanntestes Beispiel für ein solches Gremium sind unabhängige Zentralbanken: Sie haben das Ziel, für ein stabiles Preisniveau zu sorgen. Da expansive Geldpolitik kurzfristig (insbesondere vor Wahlen) genutzt werden kann, um für höhere Beschäftigung zu sorgen, erliegen Politiker häufig der Versuchung, dies zu nutzen, um ihre Wiederwahlchancen zu erhöhen. Langfristig (nach Wahlen) führt eine expansive Geldpolitik aber zu höherer Inflation, was der Wirtschaft nachhaltig schaden kann. Daher sind mehr und mehr Länder dazu übergegangen, geldpolitische Kompetenzen an nicht-gewählte Zentralbanker zu delegieren, die diesen verzerrten Anreizen nicht unterliegen.

Inzwischen werden unabhängige Gremien in vielen Politikbereichen genutzt. Für ihre Existenz sind viele Gründe 
angeführt worden, die weit über das für die Geldpolitik bedeutsame Problem der Zeitinkonsistenz ${ }^{1}$ hinausreichen. Ackerman (2000) spricht sogar von einer „new separation of powers“. Um etwas Ordnung in diesen neuen Zweig der Gewaltenteilung zu bringen, hat Vibert (2007) vorgeschlagen, sie in fünf Gruppen zu unterteilen:

1. Dienstleister: Zentralbanken, Statistikämter, Agenturen zur Entwicklung technischer Standards und Rundfunkanstalten;

2. Risikobewerter: alle Agenturen, die sich mit der Bewertung, aber auch dem Management von Risiken beschäftigen;

3. Regulierungsdurchsetzer (boundary watchers): Kartellämter und Branchenregulierer, aber auch Datenschützer. Hier sollen unter anderem die Grenzen zwischen staatlichem und privatem Sektor festgelegt werden;

4. Untersucher: Rechnungshöfe, wobei auch viele nichtgewählte Gremien einem Audit unterliegen;

5. Schiedsrichter und Whistleblower: Gremien oder Personen, durch die Entscheidungen staatlicher Ämter einschließlich jener, deren Spitzen nicht gewählt werden - angefochten werden können.

Es werden zahlreiche Beispiele für jede Kategorie genannt. Hier seien nur ein paar für Deutschland herausgegriffen. Das Bundesamt für Naturschutz (1), das Bundesamt für Risikobewertung (2), die Bundesanstalt für Finanzdienstleistungsaufsicht (BaFin) (3) und der Bundesrechnungshof (4). Für die letzte Kategorie nennt Vibert kein Beispiel aus Deutschland, aber hier könnten etwa Datenschutzbeauftragte oder Ombudsleute genannt werden.

Wie unabhängige Gremien legitimiert werden können

Seit einigen Jahren wird auch in Deutschland der Ruf nach einer evidenzbasierten Wirtschaftspolitik laut. Damit ist eine Politik gemeint, die nicht nur auf der Basis theoretischer Modelle operiert, sondern sich auf vorhandene empirische Evidenz zur Funktionsweise von Wirtschaft und Politik stützt (z.B. Buch et al., 2019). Ein Argument für unabhängige Gremien ist in diesem Zusammenhang, dass mit Experten besetzte Gremien viel besser in der La- ge sind, empirische Evidenz adäquat zu berücksichtigen als gewählte Politiker, die hingegen Spezialisten für den am Ende notwendigen Interessenausgleich und dessen gesetzliche Durchsetzung sind (Vibert, 2007).

Das Argument, dass Expertengremien Informationen besser verarbeiten und Sachentscheidungen besser treffen als Gremien, die einer Wiederwahl-Restriktion unterliegen, wurde bereits von Maskin und Tirole (2004) beschrieben. Die auch von ihnen hervorgehobene Überlegung, dass werturteilsbehaftete Politikentscheidungen in einer Demokratie weiterhin von gewählten Vertretern getroffen werden sollten, muss jedoch berücksichtigt werden, wenn man über die Konstruktion und die Kompetenzen von Expertengremien nachdenkt. ${ }^{2}$

\section{Politökonomische Überlegungen}

Wir gehen davon aus, dass Politiker (wieder-)gewählt werden wollen. Unter welchen Bedingungen könnten sie bereit sein, Kompetenzen an unabhängige Gremien zu delegieren? Voigt und Salzberger (2002) haben dazu eine Vielzahl möglicher Argumente zusammengetragen. Delegation von Kompetenz kann ein Instrument sein, um

- Verantwortung zu delegieren;

- Unsicherheit über Konsequenzen bestimmter Politiken aus dem Weg zu gehen;

- die eigene Legitimität zu erhalten;

- die eigenen langfristigen Präferenzen zu realisieren;

- die eigene Politik vor zukünftigen Veränderungen zu schützen;

- sich glaubhaft an eine bestimmte Politik zu binden;

- mehr Informationen in politische Entscheidungen einfließen zu lassen;

- den eigenen Entscheidungsaufwand zu reduzieren;

- wahlbedingte politische Entscheidungszyklen zu reduzieren.

Allerdings ist es mit Kosten verbunden, Entscheidungskompetenz von gewählten Politikern an nicht-gewählte 
Funktionsträger zu delegieren. Voigt und Salzberger (2002) nennen folgende Kategorien:

- Die Aufhebung einer von einem nicht-gewählten Gremium getroffenen Entscheidung durch die Politik kann mit erheblichem Druck auf gewählte Politiker einhergehen;

- die mit der Erledigung bestimmter Aufgaben betrauten Gremien können im Laufe der Zeit immer mehr von den Präferenzen der Politiker abweichen (delegatee drift);

- um letzteres zu verhindern, müssen möglicherweise eigene Kontrollmechanismen etabliert werden - diese sind kostenträchtig;

- die Delegation von Kompetenzen kann mit einem Transfer von Legitimität, d. h. mit zunehmender Delegitimierung gewählter Volksvertreter, einhergehen;

- wenn neue Gremien geschaffen werden, kann Unsicherheit entstehen, wer für einen Politikbereich verantwortlich ist. Daher empfiehlt sich eine präzise Aufgabenzuweisung.

Eine Entscheidung für die Delegation bestimmter Kompetenzen im Bereich der Rentenversicherung an ein unabhängiges Gremium sollte die hier genannten Nutzen- und Kostenkomponenten explizit berücksichtigen. ${ }^{3}$ Auch bei der Ausgestaltung des Gremiums selbst ist zu fragen, wie mögliche Kosten gering gehalten werden können, etwa durch eine präzise Aufgabenbeschreibung sowie ein durchdachtes Auswahlverfahren der Mitglieder.

\section{Mögliche Kompetenzen des Rentenrats}

Politiker tun sich mit Rentenreformen seit den 1970er Jahren schwer, weil es nur geringen Spielraum für punktuelle Leistungsverbesserungen gab und einen hohen Druck, die Kostenbelastung durch die Rentenleistungen im Vergleich zum Status quo ante zu senken. Sie befürchten, mit leistungskürzenden Reformen ihre Wiederwahl zu gefährden. Eine solche Rentenreform war 1989 nur möglich, weil damals ein Konsens zwischen der Regierungskoalition aus CDU und FDP sowie der starken Oppositionspartei SPD zustande kam. Im Nachgang gab es keine politische und öffentliche Diskussion, da am Tag nach der Verabschiedung des Reformgesetzes die Berliner Mauer fiel.

Es gibt nicht nur eine ganze Reihe von Argumenten, die für unabhängige Gremien sprechen, um schwierige po-

3 Für Vorüberlegungen vgl. Voigt und Wagner (2000) und Wagner (2002). litische Entscheidungen zu ermöglichen, sondern auch positive Erfahrungen aus ganz unterschiedlichen Politikfeldern. Anlass genug, über die Möglichkeit nachzudenken, einen unabhängigen „Rentenrat" einzuführen. Hier werden zunächst einige Optionen skizziert, mit welchen Kompetenzen ein unabhängiger „Rentenrat“ ausgestattet sein könnte. Die Optionen sind nach dem Einfluss sortiert: Option 1 lässt der Politik alle Möglichkeiten, während Option 5 eine weitgehende Selbstbindung der Politik impliziert.

1. Initiativrecht - der Rentenrat hat das Recht, auf Probleme hinzuweisen, die bei unveränderter Rentenpolitik entstehen würden (entspricht in etwa den Möglichkeiten des seit 1958 existierenden Sozialbeirats beim Ministerium für Arbeit und Soziales);

2. Vorschlagsrecht - der Rentenrat hat das Recht, Vorschläge zu unterbreiten (auch dies ist dem Sozialbeirat möglich);

3. Vorschlagspflicht - der Rentenrat hat die Pflicht, Vorschläge zur Lösung der identifizierten Probleme zu unterbreiten; diese Pflicht könnte mit der Pflicht verknüpft werden, dass Regierung und Parlament sich in angemessener Form zu den Vorschlägen äußern müssen;

4. Agenda-setting-Monopol - allein der Rentenrat hat die Kompetenz, Vorschläge zur Reform der jeweils gültigen Rentenregeln zu unterbreiten. Eine solche Konstruktion ist jedoch mit der Gefahr verbunden, den Reformstau weiter zu verstärken;

5. Entscheidungskompetenz - es wird ein Mechanismus etabliert, der dem Rentenrat die Kompetenz erteilt, Veränderungen im Rentensystem nicht nur vorzuschlagen, sondern in klar abgegrenzten Bereichen direkt umzusetzen (analog der Kompetenzdelegation an eine Zentralbank).

Option 3 (Vorschlagspflicht) wäre eine Verbesserung gegenüber dem Status quo, weil der Rentenrat die Politik dabei unterstützen würde, unpopuläre Entscheidungen vorzubereiten. Der Vorschlag der Rentenkommission zur Schaffung eines Alterssicherungsbeirats entspricht dieser Option, weil der Beirat verpflichtet werden soll, Parlament und Regierung Empfehlungen zu geben. Ein Rentenrat mit Vorschlagspflicht könnte hinsichtlich aller (denkbaren) Parameter einer Altersvorsorge eine Kompetenz haben oder nur hinsichtlich vom Gesetzgeber ausgewählter Parameter, wie (1) Mindestrente, (2) Rentenniveau, (3) Beitragssatz und (4) Rentenzugangsregelungen. Der Kommissionsbericht sieht vor, dass der Alterssicherungs- 
beirat Empfehlungen zu (2) und (3) gibt und - allerdings erstmals erst 2026 - eine Empfehlung zur Altersgrenze, also zu (4).

\section{Der Idealfall: ein Rentenrat mit eigener Entscheidungskompetenz}

Option 5 umzusetzen - also der Schaffung eines Rentenrats mit eigener Entscheidungskompetenz -, erscheint auf den ersten Blick abwegig. Allerdings gibt es auch in Deutschland mindestens ein Beispiel für eine Kommission mit einer sehr weitreichenden Kompetenz: Die Entscheidungen über die Festsetzung des Mindestlohns werden von der Mindestlohnkommission getroffen und qua Rechtsverordnung verbindlich gemacht. Allerdings sind Entscheidungen über die Rente mehr als nur eine einzige Sachentscheidung, weil die Rentenversicherung ein Zielbündel verfolgt und die Gewichtung der einzelnen Ziele Werturteile erfordert.

Eine besondere Herausforderung für das institutionelle Design eines Rentenrats ist die erwünschte Verschränkung zwischen Parlament und Rentenrat. Eine Verschränkung wird gewünscht, weil Entscheidungen über die Rente immer auch werturteilsbehaftet sind. Eine explizite parlamentarische Zustimmung ist für die Legitimation der Rentenpolitik unumgänglich. Eine Verschränkung ist aber auch geboten, weil dem Rentenrat weder eine Kompetenz zur Verschuldung gegeben noch inm die Entscheidung über die Höhe des Bundeszuschusses überlassen werden kann. ${ }^{4}$

Wenn allerdings - wie bisher - der Gesetzgeber die Entscheidung über die Höhe des Bundeszuschusses trifft, könnte die durch die Gründung des Rentenrats intendierte Verschiebung der Verantwortung (responsibility shift) verhindert werden: Sollten die Einnahmen aus der Rentenversicherung nicht mehr ausreichen, dürfte in der Öffentlichkeit schnell die Forderung laut werden, der Rückgang sei durch eine entsprechende Erhöhung des Bundeszuschusses auszugleichen. Der Bundeszuschuss setzt sich derzeit aus einer Reihe von Einzelposten zusammen, deren Höhe sich unter anderem nach der Lohnentwicklung und dem jeweils gültigen Beitragssatz richtet. Aber auch diese Regeln können geändert werden. Um zu verhindern, dass die Politik die Entscheidungen des Rentenrats durch entsprechende Erhöhungen des Bundeszuschusses konterkarieren kann, ist zu er-

4 Andererseits gibt es unabhängige Gremien, die mit ähnlich weitreichenden Befugnissen ausgestattet sind. So schreibt das sächsische Kulturraumgesetz nicht nur die Höhe der jährlichen Zuwendungen des Freistaats Sachsen an die Kulturräume fest, sondern macht die Entscheidungen seines Kulturbeirats auch bindend. Abweichungen sind allerdings möglich, müssen jedoch schriftlich begründet werden. wägen, dem Rentenrat die alleinige Kompetenz zu geben, Veränderungen der Berechnungsgrundlagen zu initiieren.

Ein Rentenrat mit Entscheidungskompetenz könnte analog dem Kartellamt konstruiert werden: In einem Fusionsfall kann der Wirtschaftsminister ein Fusionsverbot des Kartellamts überstimmen. Übertragen auf die Rentenpolitik: Die Entscheidungskompetenz würde zunächst einmal beim Rentenrat liegen. Sollte eine Mehrheit der Parlamentarier allerdings vom Vorschlag des Rentenrats abweichen wollen, wäre dies möglich und legitim. Tatsächlich haben die Wirtschaftsminister von ihrem Recht, ein Fusionsverbot mithilfe der Ministererlaubnis auszuhebeln, nur sehr selten Gebrauch gemacht. Durch eine intelligente Konstruktion könnte dies auch in der Rentenpolitik gelingen. Im Parlament könnte z. B. eine Zweidrittelmehrheit erforderlich sein, um Entscheidungen des Rentenrats auszuhebeln. ${ }^{5}$

\section{Mögliche Kriterien für die Ausgestaltung des Rentenrats}

Neben den Kompetenzen eines Rentenrats und den Restriktionen, unter denen er agieren soll, ist seine mögliche Zusammensetzung eine dritte zentrale Dimension. Dabei spielen die erwünschten Eigenschaften der Mitglieder, die Zahl seiner Mitglieder sowie das gewählte Ernennungsverfahren eine zentrale Rolle.

\section{Details der Aufgabendelegation}

Die Delegation von Entscheidungskompetenz an ein nicht gewähltes unabhängiges Gremium impliziert die Gefahr eines „agency drift“. Um diese Gefahr so gering wie möglich zu halten - und dem Gremium gleichzeitig eine möglichst hohe Legitimität zu geben -, ist eine Reihe von Maßnahmen denkbar:

- Ein Gesetz legt die Aufgaben des Rentenrats so präzise wie möglich fest.

- Um ein „Diener-zweier-Herren-Problem“ zu vermeiden, wird die Aufsicht über den Rentenrat einem einzigen Bundesministerium anvertraut, am Sinnvollsten dem Bundesministerium für Arbeit und Soziales.

- Damit die Öffentlichkeit die Entscheidungsprozesse des Rentenrats nachvollziehen kann, ist ein hoher Grad von Transparenz erwünscht. Diese kann durch die zeit-

5 Eine solche Institutionalisierung wäre auch eine Möglichkeit, einer sehr weitgehenden „agency drift“ vorzubeugen. 
nahe Veröffentlichung von Sitzungsprotokollen sichergestellt werden.

Schließlich verbleibt die ultimative Kompetenz bei der Regierung, wenn ein Eingriffsmechanismus analog zur Fusionskontrolle etabliert wird.

\section{Erwünschte Eigenschaften der Mitglieder}

- "Weise“ und „erfahren“ sind zwei Eigenschaften, die wünschenswert sind. Wenn vermutet wird, dass eine zentrale Konfliktlinie zwischen Beitragszahlern einerseits und Rentenempfängern andererseits verläuft, dann bräuchte man Mitglieder, die wie Schlichter bei Tarifverhandlungen wirken können. Denkbar sind ehemalige Politiker, die dabei helfen könnten, das Gremium zu erden.

- Die Mitglieder sollten über einen langen Zeithorizont verfügen. Das scheint einerseits zu implizieren, dass der Rentenrat wenig alte Mitglieder haben sollte. Andererseits kann argumentiert werden, dass ältere Mitglieder das Wohl ihrer Kinder bzw. Enkel im Blick haben und dass sie neutralere Entscheidungen treffen könnten, weil ihre eigene Rente von den getroffenen Entscheidungen nicht mehr zentral betroffen ist.

- Experten, d.h. Vertreter ganz unterschiedlicher wissenschaftlicher Disziplinen, können zur hohen Qualität der Entscheidungen eines Rentenrats beitragen, etwa Demografen, Arbeitsmarktökonomen, Versicherungswissenschaftler und Juristen.

\section{Zusammensetzung des Rentenrats}

Verschiedene Konstruktionen sind denkbar:

- ein reines Expertengremium (möglicherweise allein aus Wissenschaftlern bestehend);

- der Versuch der Abbildung gesellschaftlich relevanter Gruppen, etwa in Anlehnung an die Rundfunkräte;

- Zusammensetzung aus Vertretern der betroffenen Gruppen sowie aus einer ausreichend großen Zahl von Wissenschaftlern: Vertreter des öffentlichen Lebens könnten neben Arbeitgeber- und Arbeitnehmervertretern z. B. Delegierte der Sozialverbände und kirchlicher Hilfswerke sein. Um eine evidenzbasierte Rentenpolitik zu ermöglichen, wäre es sinnvoll, dass die Vertreter der Wissenschaft eine Mehrheit im Rentenrat stellen. Die Erfahrungen mit der Rentenkommission legen nahe, dass dem Rentenrat keine Politiker angehören sollten, die noch aktive Parteipolitik betreiben und ein Interesse an einer Wiederwahl haben.

\section{Größe des Rentenrats}

Die Zahl der Mitglieder wird im Wesentlichen von der Entscheidung bestimmt, wie der Rentenrat zusammengesetzt werden soll. Allerdings hängt die Arbeitsfähigkeit eines Gremiums auch von seiner Größe ab. Bei einer sehr hohen Mitgliederzahl ist der individuelle Anreiz, sich intensiv auf die Diskussionen im Rentenrat vorzubereiten, gering, weil das eigene Stimmengewicht klein ist. Jedoch würde ein sehr kleiner Rentenrat weniger Ideen produzieren und weniger Argumente systematisch abwägen. Eine mittlere Größe ( zwölf bis 15 Mitglieder) erscheint deshalb sinnvoll.

Unabhängig von der gewählten Mitgliederzahl des Rentenrats ist die Errichtung einer permanenten Geschäftsstelle mit wissenschaftlicher Kompetenz nicht nur sinnvoll, sondern notwendig, um dem Rat Unabhängigkeit von Berechnungen und Prognosen des zuständigen Ministeriums und der Rentenversicherung zu geben.

\section{Ernennungsverfahren}

Das Verfahren, mit deren Hilfe Mitglieder des Rentenrats ernannt werden, hängt von der Gewichtung der verfolgten Ziele ab. Wenn unterstellt wird, dass die Einrichtung eines Rentenrats das Ziel hat, evidenzbasierte Politik zu stärken und die Rentenversicherung damit zukunftsfest zu machen, dann ist es wichtig, dass die wissenschaftlichen Mitglieder Experten sind - und nicht verkappte Interessenvertreter. Als Vorbild für ein Ernennungsverfahren könnte der Wissenschaftsrat dienen. Die Mitglieder der wissenschaftlichen Kommission des Wissenschaftsrats werden nach gemeinsamem Vorschlag der Deutschen Forschungsgemeinschaft, der Max-Planck-Gesellschaft, der Hochschulrektorenkonferenzen, der HelmholtzGemeinschaft, der Fraunhofer-Gesellschaft und der Leibniz-Gemeinschaft vom Bundespräsidenten berufen. Dieses Verfahren hat sich bewährt, zumal das Gremium ergänzt wird um , anerkannte Persönlichkeiten des öffentlichen Lebens", die von den üblichen Interessenverbänden nominiert werden (formal nominiert werden sie „auf gemeinsamen Vorschlag der Bundesregierung und der Landesregierungen"). ${ }^{6}$

\section{Dauer der Mitgliedschaft}

Die Mitgliedschaft im Rentenrat sollte es den Mitgliedern ermöglichen, sich mit den Aufgaben und der Arbeitsweise des Gremiums vertraut zu machen und deshalb eine ge-

6 Vgl. Artikel 4 des „Verwaltungsabkommens zwischen Bund und Ländern über die Errichtung eines Wissenschaftsrates vom 5. September 1957 (in der ab 1. Januar 2008 geltenden Fassung)“, (2008). 
wisse Mindestdauer nicht unterschreiten. Allerdings sollte die Mitgliedschaft auch nicht zu lang sein. Eine Ernennung für (zunächst) sechs Jahre erscheint sinnvoll.

Die Möglichkeit der Wiederernennung gilt gemeinhin als problematisch für die Unabhängigkeit eines Gremiums: Ein Mitglied, das eine Wiederernennung wünscht, hat Anreize, die Präferenzen der darüber Entscheidenden ernst zu nehmen, um die Chancen einer erneuten Ernennung zu wahren. Für das skizzierte Ernennungsverfahren in Anlehnung an das Verfahren des Wissenschaftsrats scheint das jedoch kein Problem zu sein. Die Möglichkeit einer einmaligen Wiederernennung kann deshalb erwogen werden. Ein Vorteil dieser Regelung bestünde darin, dass sehr passive Mitglieder nach sechs Jahren nicht wieder ernannt werden müssten.

Somit ist eine durchschnittliche Zugehörigkeitsdauer zum Rentenrat von weniger als zwölf Jahren zu erwarten. Um sicherzustellen, dass der Rentenrat immer wieder von neuen Ideen profitiert - genauso wie von neuen wissenschaftlichen Einsichten - könnte ein gestaffeltes Ernennungsverfahren installiert werden. Abhängig von der Gesamtmitgliederzahl könnten alle zwei bis vier Jahre jeweils zwei bis vier neue Mitglieder ernannt werden.

\section{Entschädigung für die Mitgliedschaft im Rentenrat}

Die nicht-wissenschaftlichen Mitglieder des Rentenrats wären Vertreter relevanter gesellschaftlicher Gruppen und bedürfen keiner speziellen Bezahlung. Die wissenschaftlichen Mitglieder des Rentenrats dürften ganz überwiegend etablierte Hochschullehrer sein. Honorare als Anreiz für eine aktive Mitgliedschaft haben vermutlich nur eine sehr begrenzte Wirkung. Stattdessen könnte die Mitgliedschaft im Rentenrat etwa mit einer ganzen oder einer Zwei-Drittel-Stelle für wissenschaftliche Mitarbeiter bzw. Doktoranden honoriert werden. Mit einer solchen Art der Entschädigung würde gleichzeitig der wissenschaftliche Nachwuchs gefördert.

\section{Rentenrat kann Renten zukunftsfest machen}

Nicht nur unter Experten besteht Einigkeit darüber, dass unser Rentensystem dringend reformiert werden muss.
Politiker fürchten allerdings, sich durch eine solche Reform unbeliebt zu machen und so ihre Wiederwahl zu gefährden. Um notwendige Reformen zu erleichtern, die von der gerade beendeten Rentenkommission nur skizziert wurden, aber mangels politischer Einigkeit nicht im Detail spezifiziert werden konnten, wird in diesem Beitrag die Gründung eines mit weitgehenden Kompetenzen ausgestatteten Rentenrats empfohlen. Der von der Rentenkommission empfohlene Alterssicherungsbeirat ist ohne Zweifel ein Schritt in die richtige Richtung. Der hier entwickelte Vorschlag geht noch einen Schritt weiter. Er ist damit auch ein Beitrag zu einer Diskussion über die Zukunft unseres Rentensystems, die jetzt geführt werden muss.

\section{Literatur}

Ackerman, B. (2000), The new separation of powers, Harvard Law Review, 633-729.

Buch, C. M., K. Patzwaldt, T. T. Riphahn und E. Vogel (2019), Verstehen - Entwickeln - Testen - Verbessern: Rahmenbedingungen für evidenzbasierte Politik, Wirtschaftsdienst, 99(2), 106-112, https://www. wirtschaftsdienst.eu/inhalt/jahr/2019/heft/2/beitrag/verstehen-entwickeln-testen-verbessern-rahmenbedingungen-fuer-evidenzbasierte-politik.html (1. April 2020).

Epstein, D. und S. O‘Halloran (1999), Delegating powers: A transaction cost politics approach to policy making under separate powers, Cambridge University Press.

Majone, G. (1996), Temporal consistency and policy credibility: why democracies need non-majoritarian institutions, Working Paper RSC, Nr. 96/57, European University Institute.

Maskin, E. und J. Tirole (2004), The politician and the judge: Accountability in government, American Economic Review, 94(4), 1034-1054.

Regierungskommission „Verlässlicher Generationenvertrag“ (2020), Bericht, https://www.verlaesslicher-generationenvertrag.de/ aktuelles/2020-03-27-kommission-verlaesslicher-generationenvertrag-uebergibt-ihren-bericht/ (3. April 2020).

Verwaltungsabkommen zwischen Bund und Ländern über die Errichtung eines Wissenschaftsrates vom 5. September 1957 (in der ab 1. Januar 2008 geltenden Fassung) (2008), https://www.wissenschaftsrat.de/ DE/Ueber-uns/Grundsatzdokumente/grundsatzdokumente_node.h tml;jsessionid=923F71D3043BA63C61464A91AC93500C.delivery2master (1. April 2020).

Vibert, F. (2007), The rise of the unelected: democracy and the new separation of powers, Cambridge University Press.

Voigt, S. und G. G. Wagner (2000), Zur Reform der Rentenversicherung ein konstitutionenökonomischer Vorschlag, Zeitschrift für Wirtschaftspolitik, 49(3), 235-249.

Voigt, S. und E. M. Salzberger (2002), Choosing not to choose: When politicians choose to delegate powers, Kyklos, 55(2), 289-310.

Wagner, G. G. (2002), Sozialpolitik und Politikberatung - Am Beispiel der regierungsamtlichen Beratung der Altersvorsorge, in U. Fachinger, $\mathrm{H}$. Rothgang und H. Viebrok. (Hrsg.), Die Konzeption sozialer Sicherung Festschrift für Winfried Schmähl zum 60. Geburtstag, 143-153.

Title: More Courage for Pension Policy! A Proposal

Abstract: There is a broad consensus that Germany's pension system needs substantial reform. However, politicians shy away from this because they are afraid that it will cost them re-election. Here, an independent 'pension council' with far-reaching competences is proposed as a possible solution.

JEL Classification: H55, J14, J32, K30 